\title{
Sex Differences in the Regulation of Vasopressin and Oxytocin Secretion in Bile Duct-Ligated Rats
}

\author{
Kirthikaa Balapattabi ${ }^{\text {a }}$ Joel T. Little ${ }^{\text {a }}$ Martha E. Bachelor ${ }^{\text {a }}$ \\ Rebecca L. Cunningham ${ }^{b}$ J. Thomas Cunningham ${ }^{a}$

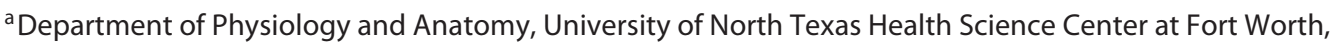 \\ Fort Worth, TX, USA; ' Department of Pharmaceutical Sciences, School of Pharmacy, University of North Texas \\ Health Science Center at Fort Worth, Fort Worth, TX, USA
}

\section{Keywords}

Ovariectomy · Adrenal · Estradiol and liver failure

\begin{abstract}
Introduction: Hyponatremia due to elevated arginine vasopressin (AVP) secretion increases mortality in liver failure patients. No previous studies have addressed sex differences in hyponatremia in liver failure animal models. Objective: This study addressed this gap in our understanding of the potential sex differences in hyponatremia associated with increased AVP secretion. Methods: This study tested the role of sex in the development of hyponatremia using adult male, female, and ovariectomized (OVX) female bile duct-ligated (BDL) rats. Results: All BDL rats had significantly increased liver to body weight ratios compared to sham controls. Male $B D L$ rats had hyponatremia with significant increases in plasma copeptin and FosB expression in supraoptic AVP neurons compared to male shams (all $p<0.05 ; 5-7$ ). Female BDL rats did not become hyponatremic or demonstrate increased supraoptic AVP neuron activation and copeptin secretion compared to female shams. Plasma oxytocin was significantly
\end{abstract}

karger@karger.com www.karger.com/nen

Karger $\stackrel{\text { ' }}{5}$

GOPEN ACCESS
(C) 2020 The Author(s)

Published by S. Karger AG, Basel

This article is licensed under the Creative Commons AttributionNonCommercial-NoDerivatives 4.0 International License (CC BYNC-ND) (http://www.karger.com/Services/OpenAccessLicense). Usage and distribution for commercial purposes as well as any distribution of modified material requires written permission. higher in female $\mathrm{BDL}$ rats compared to female sham $(p<$ $0.05 ; 6-10)$. This increase was not observed in male BDL rats. Ovariectomy significantly decreased plasma estradiol in sham rats compared to intact female sham $(p<0.05 ; 6-10)$. However, circulating estradiol was significantly elevated in OVX BDL rats compared to the OVX and female shams $(p<$ $0.05 ; 6-10)$. Adrenal estradiol, testosterone, and dehydroepiandrosterone (DHEA) were measured to identify a possible source of circulating estradiol in OVX BDL rats. The OVX BDL rats had significantly increased adrenal estradiol along with significantly decreased adrenal testosterone and DHEA compared to OVX shams (all $p<0.05 ; 6-7$ ). Plasma osmolality, hematocrit, copeptin, and AVP neuron activation were not significantly different between OVX BDL and OVX shams. Plasma oxytocin was significantly higher in OVX BDL rats compared to OVX sham. Conclusions: Our results show that unlike male BDL rats, female and OVX BDL rats did not develop hyponatremia, supraoptic AVP neuron activation, or increased copeptin secretion compared to female shams. Adrenal estradiol might have compensated for the lack of ovarian estrogens in OVX BDL rats.

(C) 2020 The Author(s)

Published by S. Karger AG, Basel

J. Thomas Cunningham

Department of Physiology and Anatomy

EAD-318B, UNT Health Science Center

3500 Camp Bowie Blvd, Fort Worth, TX 76107 (USA)

Tom.Cunningham@unthsc.edu 


\section{Introduction}

Hyponatremia is a frequent complication observed in liver failure patients [1-3]. Hyponatremia is currently defined as a serum sodium level of less than $135 \mathrm{mEq} / \mathrm{L}$ [1]. The primary cause of hyponatremia during liver failure or heart failure is inappropriate arginine vasopressin (AVP) secretion $[1,4]$. Portal hypertension and the associated systemic vasodilation lead to activation of neurohumoral mechanisms in order to maintain effective circulating volume, which include increased AVP secretion [5-8]. However, sustained AVP secretion is maintained despite decreased plasma osmolality $[4,9,10]$. Increased AVP secretion contributes to ascites, seizures, pulmonary, and cerebral edema resulting in increased mortality of liver failure patients $[1,4,11-14]$. The management of hyponatremia is challenging as conventional therapy including fluid restriction and loop diuretics are frequently inefficacious $[2,4,8$, 13-15]. In addition to our limited understanding of the mechanisms underlying inappropriate AVP secretion, sex differences in the development of hyponatremia during liver failure have not been extensively investigated.

The prevalence of liver disease is higher in men and increases in women after menopause, and one study indicates that estrogen may reduce carbon tetrachlorideinduced hepatic fibrosis in female rats [16-18]. This suggests protective effects of estrogen but the role of ovarian hormones in the progression of liver failure, but the regulation of AVP release in this disease modality remains to be understood [19-21]. Systemic oxytocin (OXT) and AVP are primarily produced by magnocellular neurosecretory cells (MNCs) located in the supraoptic nucleus (SON) and the paraventricular nucleus of hypothalamus [22-25]. Several studies have established that AVP MNCs express ER $\beta$ while the circumventricular organs express $\mathrm{ER} \alpha$ and project efferent fibers to the PVN and SON [19, 20,26-28], indicating the potential for the regulation of MNCs by estrogen. Previous studies have shown that estrogen can inhibit AVP release in vitro $[18,21,26]$ and the same studies show that estrogen inhibits OXT release in vitro as well.

In addition to sexual dimorphism in AVP secretion [29], previous studies have shown that the pituitary levels of vasopressin were maximal in proestrus, declined during estrus, and were lowest during metestrus in cycling female rats [30] and the concentration of AVP in PVN and SON was significantly lower in animals during estrus [31]. No previous studies report sex differences in development of hyponatremia and AVP secretion during liver cirrhosis. A better understanding of mechanisms under- lying sex difference in AVP secretion associated with liver cirrhosis may open new avenues of treatment for hyponatremia and a better understanding of sex differences related to body fluid balance. The aim of this study is to investigate potential sex differences in dilutional hyponatremia and the effect of ovarian hormones on AVP secretion. Chronic bile duct ligation (BDL), a model of liver cirrhosis that develops hyponatremia, was used in this study $[10,32,33]$. This is the first study to address sex differences in hyponatremia in this liver disease model.

\section{Materials and Methods}

\section{Animals}

Adult male (450-500 g) and female (250-300 g) Sprague-Dawley rats (Charles River, Wilmington, MA, USA) were used in the experiments for this study. Experimental protocols involving animals were approved by the UNT Health Science Center IACUC and conducted in accordance to the National Institute of Health Guide for the Care and Use of Laboratory Animals. All animals were housed in a temperature $\left(24-26^{\circ} \mathrm{C}\right)$ and humidity $(40-60 \%)$ controlled environment with ad libitum access to food (Teklad LM-485 Rat Sterilizable Diet with 0.3\% Sodium; Envigo, Somerset, NJ, USA) and water unless otherwise indicated. Rats were individually housed due to the use of survival surgery and individual fluid intake measurements in the protocol. Survival surgeries were conducted using aseptic techniques. All rats were given procaine penicillin $\mathrm{G}(30,000 \mathrm{U}$, s.c.) and non-steroidal anti-inflammatory drug, carprofen (Rimadyl, $2 \mathrm{mg}$ p.o.), was given before and after surgery for pain management.

\section{Bilateral Ovariectomy}

Prior to ovariectomy, female rats were anaesthetized with isoflurane $(2-3 \%)$. The left side of the abdomen was shaved, cleaned, and incision was created. Ovary and uterine horn were identified and exposed. The ovary was cut by gently pulling it out by grabbing the surrounding adipose tissue to prevent detachment of a small piece of ovary, which may fall into the abdominal cavity where it may be replanted to carry on its normal function [34]. The fallopian tube was cauterized, and the remaining tissue was put back into the abdominal cavity. The incision was closed with absorbable antibiotic sutures. The procedure was repeated on the right side to remove the other ovary [35]. Two weeks later, the rats received either BDL or sham ligation surgery.

\section{BDL Surgery}

The rats were anaesthetized with isoflurane (2-3\%). The abdomen was shaved and cleaned. A midline abdominal incision was performed, and the common bile duct was isolated and cauterized between two ligatures as previously described $[32,36]$. Visual inspection of ascetic fluid accumulation in the peritoneal cavity was performed daily after surgery. Any rat showing morbidity or ascites that appeared to be greater than $10 \%$ of the body weight was euthanized. Sham rats received the same surgical procedure except their bile duct was not cauterized. Liver to body weight ratio was determined for every rat at the end of the study to verify the development of liver cirrhosis. 
Metabolic Cage Study

After 2 weeks of recovery from BDL or sham ligation surgery, the rats were moved into metabolic cages (Lab Products, Seaford, DE, USA) to measure food intake, fluid intake, and urine excretion every day. The rats were provided with ad libitum access to food and water for 14 days to record various parameters as previously described [25]. Food intake was measured by filling the food containers up to a predetermined weight of ground chow (Teklad Diets, Madison, WI, USA) in grams. After $24 \mathrm{~h}$, the food was reweighed, and the amount consumed was calculated by the difference. Fluid intake was measured by filling the bottles with a known amount of water in grams and subtracting the remaining weight $24 \mathrm{~h}$ later. Mineral oil was added to the urine collection vials to prevent evaporation of water from urine. Urine excretion volume was recorded, and urine samples were collected for measuring daily sodium excretion.

\section{Estrous Cycling in Female Rats}

Evaluation of vaginal cytology in the female rats on the day of decapitation was performed based on the relative abundance of each cell type in the smear as previously described [37]. Vaginal smears were collected with micropipette by inserting the tip of the pipette into the vaginal orifice with relatively shallow penetration. Distilled water $(0.2-0.25 \mathrm{~mL})$ was used for flushing without markedly distorting the cells to impair identification. The fluid was collected and placed on a glass slide. Smears were evaluated immediately, fresh and unstained. A standard laboratory light microscope (Olympus Corporation, Center Valley, PA, USA) was used to evaluate the sample at a total magnification of $\times 40$. Changes in vaginal epithelial cell structure were documented to establish the stage of the estrous cycle for each female rat.

\section{Plasma Measurements}

Rats were anesthetized with inactin (thiobutabarbital sodium salt hydrate; $100 \mathrm{mg} / \mathrm{kg}$ i.p.) and trunk blood was collected following decapitation. A 5-6 mL sample of blood was collected in Vacutainer tubes containing the anticoagulant, EDTA $(12 \mathrm{mg})$ and the proteinase inhibitor, aprotinin $(0.6 \mathrm{TIU} / \mathrm{mL}$ of blood; Phoenix Pharmaceuticals Inc., Burlingame, CA, USA). The samples were centrifuged at $1,600 \mathrm{~g}$ for $15 \mathrm{~min}$ at $4{ }^{\circ} \mathrm{C}$. Plasma was collected from each sample for measuring copeptin (CPP), OXT, and estradiol concentrations. CPP is widely used as biomarker for AVP secretion [38]. CPP and estradiol concentrations were measured in plasma samples by using specific ELISAs according to the manufacturer's instructions (CPP: MBS724037, MyBioSource Inc., San Diego, CA, USA; estradiol: RTC009R, BioVendor Research and Diagnostic Products, Asheville, NC, USA). For OXT measurements, an aliquot of plasma was used to extract peptides by solidphase extraction using C-18 SEP-Column (Phenomenex, Torrance, CA, USA). The elutes from the column were subjected to vacuum centrifugal concentration and were used to measure circulating OXT with specific ELISA (EK-051-01; Phoenix Pharmaceuticals). Four-parameter logistic analysis (4-PL) was performed to quantify the concentrations of CPP, OXT, and estradiol.

A separate 1- to 2-mL sample of blood was collected in a $2-\mathrm{mL}$ microcentrifuge tube and prepared for measuring plasma osmolality and hematocrit as previously described [25, 39]. Two heparinized capillary tubes (Fisher Scientific, Hampton, NH, USA) were filled with whole blood for measuring hematocrit using MicroHematocrit capillary tube reader (Lancer, St. Louis, MO, USA).
The remaining blood sample was centrifuged at $1,600 \mathrm{~g}$ for $5-10$ min, and plasma was collected to measure plasma osmolality using vapor pressure osmometer (Wescor, Logan, UT, USA).

\section{Immunohistochemistry}

Four weeks after BDL or sham surgery, rats were deeply anesthetized with inactin (thiobutabarbital sodium salt hydrate; $100 \mathrm{mg} / \mathrm{kg}$, i.p.), perfused transcardially with phosphate-buffered saline (PBS) followed by $4 \%$ paraformaldehyde in PBS. After the perfusion, brains were extracted from the skull and placed in 30\% sucrose until dehydrated. The brains were serial sectioned at a thickness of $40 \mu \mathrm{m}$ using a cryostat. Sections were processed for FosB and AVP using goat polyclonal FosB (Santa Cruz Biotechnology, Cat\# sc-48-G, Santa Cruz, CA, USA; 1:1,000), guinea pig polyclonal Anti-( $\left.\operatorname{Arg}^{8}\right)-\mathrm{VP}(1$ : 1,000; Cat\# T-5048, Peninsula Labs, San Carlos, CA, USA). Sections were incubated in primary antibodies for 2 days at $4{ }^{\circ} \mathrm{C}$. Following primary antibody incubation, sections were rinsed with PBS, followed by sequential incubation in respective secondary antibodies against host species. Biotinylated anti-goat secondary (1:1,000, Jackson ImmunoResearch Laboratories Inc., West Grove, PA, USA) was used for FosB to visualize using 3,3'-diaminobenzidine hydrochlorides. The Cy3 fluorophores (Cy3-conjugated anti-guinea pig IgG; 1:10,000; Cat\# 706-165-148, Jackson ImmunoResearch Laboratories) was used for AVP. Sections were rinsed again in PBS, mounted on gelatin-coated slides, and coverslipped with Vectashield (Vecta Labs, Burlingame, CA, USA) mounting media. Sections were examined for co-localization of the FosB with AVP immunofluorescence to determine the activation of AVP neurons.

\section{Adrenal Gland Steroid Measurements}

Four weeks after BDL or sham surgery, the adrenal glands from ovariectomized (OVX) female rats were collected and flash frozen using dry ice. The frozen $\left(-80^{\circ} \mathrm{C}\right)$ adrenal tissue was thawed, weighed, and homogenized using acetonitrile. The steroids were extracted from tissue samples by organic phase extraction using acetonitrile to solubilize the steroid and hexane to remove fat and lipid that may be present in the sample. The steroid separated in acetonitrile phase was evaporated to dryness using vacuum centrifugal concentrator [40]. The dried extracted samples were diluted in ethanol: assay buffer mixture $(1: 10)$. The reconstituted samples were used to measure dehydroepiandrosterone (DHEA), testosterone, and estradiol concentration using specific ELISAs according to the manufacturer's instructions (DHEA: Enzo Life Sciences Inc., Farmingdale, NY, USA; testosterone: BioVendor Research and Diagnostic Products). Four-parameter logistic analysis (4-PL) was performed to quantify the concentrations of DHEA, testosterone, and estradiol.

\section{Experimental Groups and Statistical Analysis}

The male, female, and OVX female rats were subdivided into two groups in these experiments: (1) sham ligation and (2) BDL. The female rats used in the study were in different stages of the estrous cycle, which contributed to some of the variability in certain endpoint measurements obtained from the female groups but did not affect the overall outcome of the analyses. The adrenal steroid concentration data from OVX sham and BDL rats was analyzed using Student's $t$ test. All other data were analyzed using two-way ANOVA with treatment (sham vs. BDL) as first factor and sex (male vs. female vs. OVX) as second factor followed by Bonferroni post hoc comparisons using Sigma Plot 12.0. Figures 


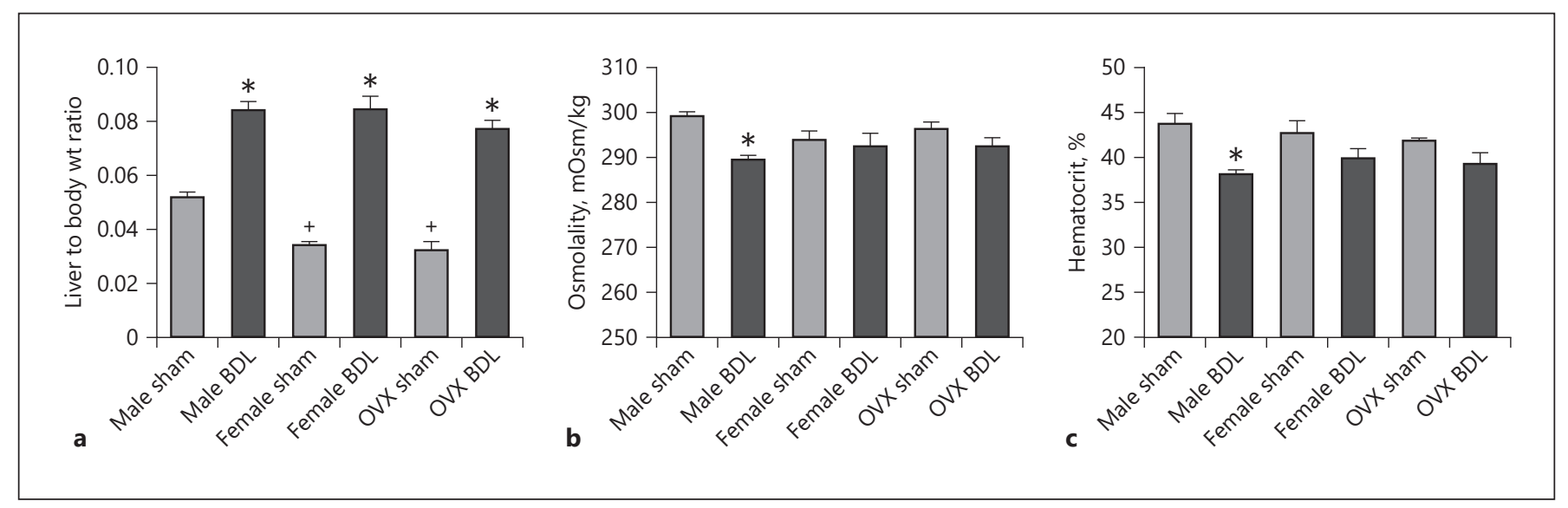

Fig. 1. Effect of BDL in male, female, and OVX rats on liver to body weight ratio (a), plasma osmolality (b), and hematocrit values (c). Data are mean \pm SEM. ${ }^{*} p<0.05$ vs. respective sham. ${ }^{+} p<0.05$ vs. respective male group. Groups: male sham $(n=6)$; male BDL $(n=6)$; female sham $(n=6)$; female BDL $(n=6)$; OVX sham $(n=8)$; and OVX BDL $(n=10-11)$

were assembled using the "magick" package in RStudio. The group sizes were determined by power analysis and effect size calculated from our previously published work $[2,39,41]$ and preliminary data using Sigma plot 12.0. The minimal number per group for each experiment was decided to have an appropriately powered study with the effect size of approximately 0.7 (70\%).

\section{Results}

\section{Liver to Body Weight Ratio}

The liver to body weight ratio was measured 4 weeks after $\mathrm{BDL} /$ sham ligation surgery as an index of liver cirrhosis $[10,32]$. The liver to body weight ratio was significantly increased in male, female, and OVX BDL rats compared to their respective sham-ligated groups. Two-way ANOVA indicated significant difference among the groups $(F(2,45)=3.36, p<0.05)$. Bonferroni post hoc analysis indicates that the ratios were significantly higher in male BDL rats compared to the male sham rats (Bonferroni $t=5.703$; $p<0.001$; Fig. 1a). Similar increase was observed in female and OVX BDL rats (female: Bonferroni $t=11.517, p<0.001$; OVX: Bonferroni $t=9.995, p<$ 0.001 ; Fig. 1a) compared to their respective sham rats. The liver to body weight ratios were not significantly different between male, female, and OVX BDL groups. This data shows that there was no sex difference in the development of liver disease with the BDL.

\section{Plasma Osmolality and Hematocrit Measurements}

Plasma osmolality and hematocrit were measured 4 weeks after BDL/sham ligation surgery. The decrease in plasma osmolality and hematocrit values in BDL rats indicates development of hyponatremia. The statistical analysis using two-way ANOVA showed that the BDL was associated with significant differences between some of the groups (osmolality: $F(1,44)=8.671, p<0.05$; hematocrit: $F(1,46)=16.754, p<0.05)$. The sex did not significantly affect basal osmolality and hematocrit.

The male BDL rats had significantly lower plasma osmolality and hematocrit values as compared to male sham rats (osmolality: Bonferroni $t=2.890 ; p<0.05$; hematocrit: Bonferroni $t=3.096 ; p<0.05$; Fig. 1b, c). Similar decreases were not observed in intact female BDL and OVX BDL rats compared to their respective sham groups (Fig. 1b, c). Unlike male BDL rats, female and OVX BDL rats did not develop hyponatremia with liver cirrhosis.

\section{Fluid Intake, Urine Excretion, Food Intake, and Body Weight}

Fluid intake, urine excretion, and food intake were measured daily for 14 days beginning 2 weeks after BDL or sham ligation surgery. Two-way ANOVA revealed a significant interaction indicating that fluid intake was affected by BDL and sex (treatment $\times$ sex: $F(39,351)=18.44$, $p<0.001$; Fig. 2a). Post hoc comparisons between the factors revealed that BDL significantly increased fluid intake in all the BDL rats compared to their respective sham (male: Bonferroni $t=4.942, p<0.05$; female: Bonferroni $t=5.271, p<0.05$; and OVX: Bonferroni $t=4.942, p<$ $0.05)$. Also, fluid intake was significantly higher in both female BDL and OVX BDL rats compared to the male BDL group (female: Bonferroni $t=5.271, p<0.05$; OVX: 
Fig. 2. Effects of BDL in male, female, and OVX rats on average daily fluid intake (a) and average daily urine excretion (b). Data are mean \pm SEM. ${ }^{*} p<0.05$ vs. respective sham. ${ }^{+} p<0.05$ vs. respective male group. Groups: male sham $(n=6)$; male BDL $(n=$ $6)$; female sham $(n=6)$; female BDL $(n=$ 6); OVX sham $(n=6)$; and OVX BDL $(n=$ $10)$.
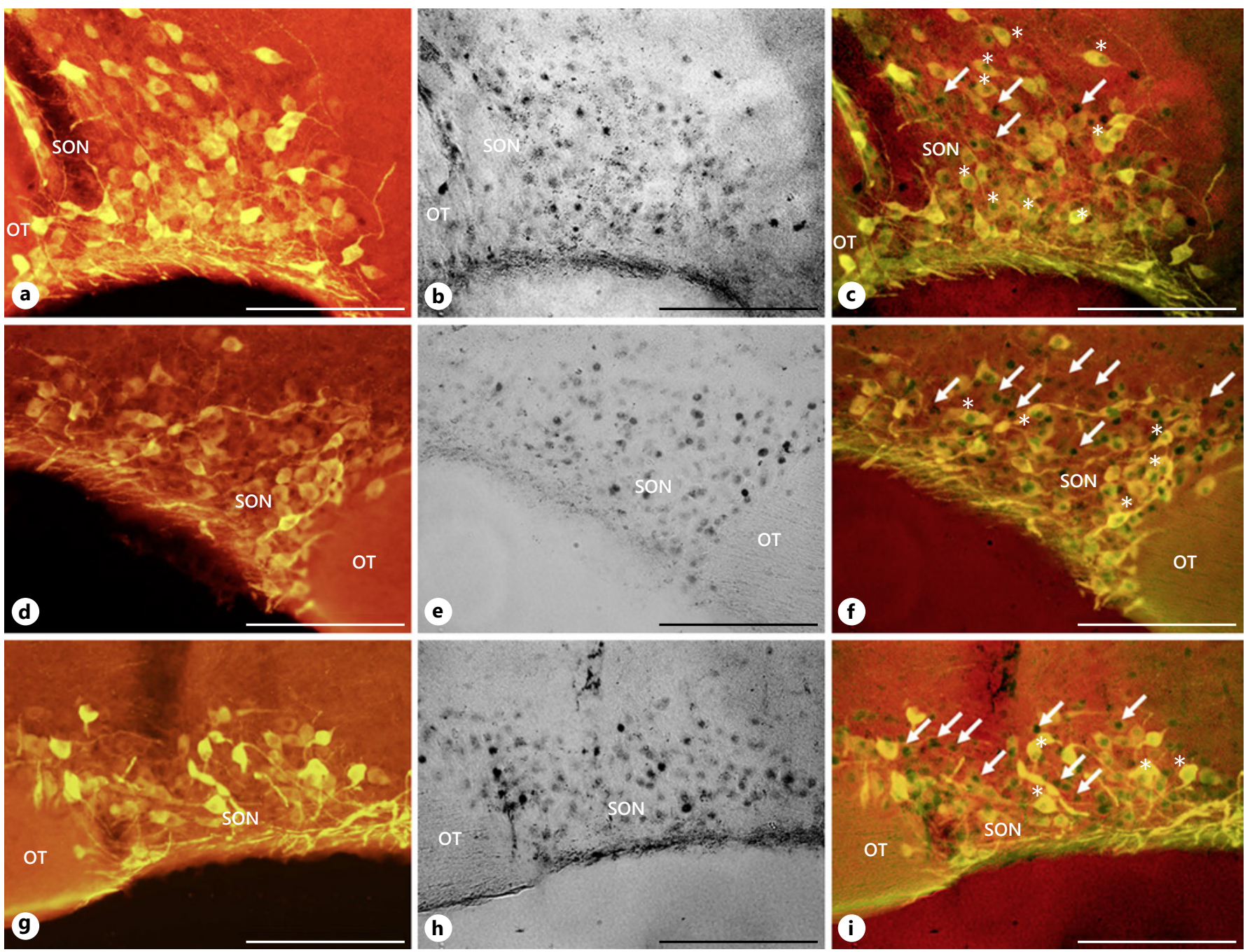

Fig. 3. Immunohistochemistry in the SON. a AVP staining in male BDL rats. b FosB staining in the same SON sections. c Double labelling of FosB and AVP obtained from the merge of A and B. $\mathbf{d}$ AVP staining in female BDL rats. e FosB staining in female BDL rats. $f$ Double labelling of FosB and AVP obtained from the merge of $\mathbf{d}$ and e. $\mathbf{g}$ AVP staining in OVX BDL rats. $\mathbf{h}$ FosB staining in
OVX BDL rats. i Double labelling of FosB and AVP obtained from the merge of $\mathbf{g}$ and $\mathbf{h}$. Merged images $(\mathbf{c}, \mathbf{f}$, and $\mathbf{i})$ : pseudocolored yellow indicates AVP and pseudocolored green indicates FosB. Asterisks indicate co-localization of FosB and AVP; arrows indicate non-colocalized neurons. 


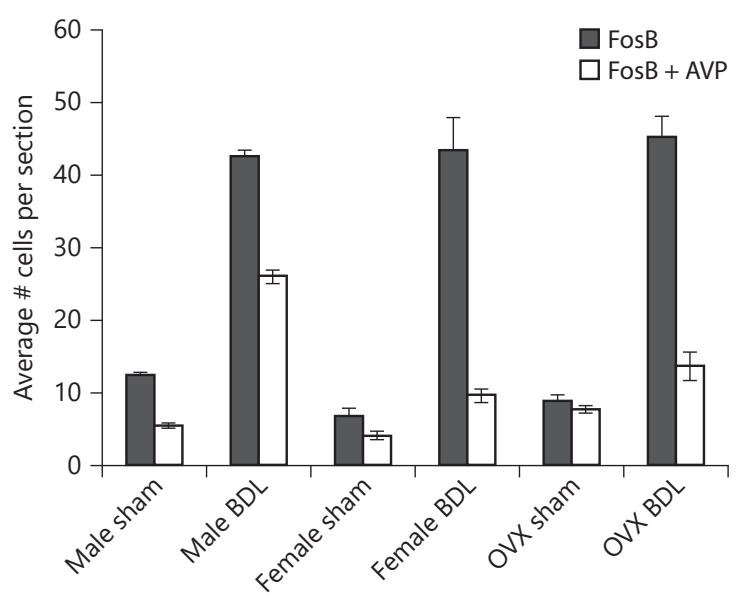

Fig. 4. Quantification of the average number of neurons positive for FosB and FosB+AVP in male sham, male BDL, female sham, female BDL, OVX sham, and OVX BDL. Data are mean \pm SEM. ${ }^{*} p<0.05$ vs. respective sham. ${ }^{+} p<0.05$ vs. respective male group. Groups: male sham $(n=6)$; male BDL $(n=6)$; female sham $(n=$ $6)$; female BDL $(n=9)$; OVX sham $(n=6)$; and OVX BDL $(n=7)$.

Bonferroni $t=4.942, p<0.05)$. A significant interaction between the factors were observed in the volume of urine excreted (treatment $\times \operatorname{sex} F(39,351)=24.419, p<0.05$; Fig. 2b). The average daily urine volume significantly increased in all the BDL groups compared to their respective sham rats (Bonferroni $t$ tests, $p<0.05$ ). Post hoc comparisons show that the urine excretion was significantly higher in female and OVX BDL rats compared to the male BDL group. The food intake and body weight were not significantly different among the groups, and increase was not significantly different among the four groups.

\section{FosB and AVP Expression in SON Neurons}

Staining for FosB was measured in the SON of male, female, and OVX rats after sham and BDL surgeries (Fig. 3b, e, h). FosB in the SON contributes to changes in gene expression that promotes development of hyponatremia in this animal model [32]. The neurons were double labeled with AVP (Fig. 3a, d, g) to determine if FosB expression was observed in AVP neurons (Fig. 3c, f, i). The numbers of FosB-positive neurons in SON was increased in male, female, and OVX BDL rats compared to their respective shams. In male BDL rats, $61 \%$ of the FosB-positive cells were vasopressinergic compared with $14 \%$ in sham-ligated controls. FosB staining was evident in AVP neurons in male BDL rats, whereas in female and
OVX BDL rats the FosB was not co-localized in AVP neurons. The $24 \%$ of the FosB-positive neurons were also AVP positive in female BDL rats compared with $19 \%$ in female sham rats. In OVX BDL rats, $29 \%$ of the FosBpositive neurons were colocalized with AVP compared with $18 \%$ in female sham rats. The number of FosB- and AVP-positive neurons in female and OVX BDL rats was not significantly higher as compared to the female shams (Fig. 4).

\section{Plasma CPP Concentration}

Plasma CPP concentration was measured 4 weeks after $\mathrm{BDL}$ or sham ligation surgery. The statistical analysis using two-way ANOVA showed a significant effect of BDL among the groups $(F(1,32)=13.219, p<0.05)$. The sex of the animal significantly affected circulating CPP $(F(2$, $32)=40.489 ; p<0.05)$. The plasma CPP concentration was significantly higher in both female groups compared to the male groups (Bonferroni $t$ tests, all $p<0.05$ ). The concentration of CPP in male BDL rats was significantly greater compared to the male sham rats (Bonferroni $t=$ 3.096; $p<0.05$; Fig. 5a). In contrast to the males, BDL was not associated with significantly higher circulating plasma CPP concentrations in female BDL rats compared to the female shams (Fig. 5a).

Circulating CPP was significantly different in OVX sham rats compared to male and female sham rats. Also, the CPP concentration in OVX BDL rats was significantly different compared to female rats but not to male rats. Similar to intact female rats, the OVX BDL rats did not have significantly greater plasma CPP concentration as compared to the OVX sham rats (Bonferroni $t$ tests, all $p<0.05$; Fig. 5a). These data indicate sex differences in basal circulating $\mathrm{CPP}$ concentration along with the difference in their response to BDL.

\section{Plasma OXT Concentration}

Plasma samples collected 4 weeks after BDL/sham ligation surgery were used for OXT measurements. The two-way ANOVA analysis shows that plasma OXT was significantly influenced by BDL and the sex of the animal (surgical treatment $(F(1,31)=44.448, p<0.05$; sex $F(2$, $31)=3.628, p<0.05)$. Plasma OXT concentration was not significantly higher in male BDL rats compared to shamligated males, whereas female BDL rats had significantly higher circulating OXT compared to sham-ligated females (Bonferroni $t=5.242 ; p<0.001$; Fig. $5 b$ ). Also, plasma OXT concentration was significantly higher in female $\mathrm{BDL}$ rats compared to the male BDL group (Bonferroni $t=3.306 ; p<0.05)$. 


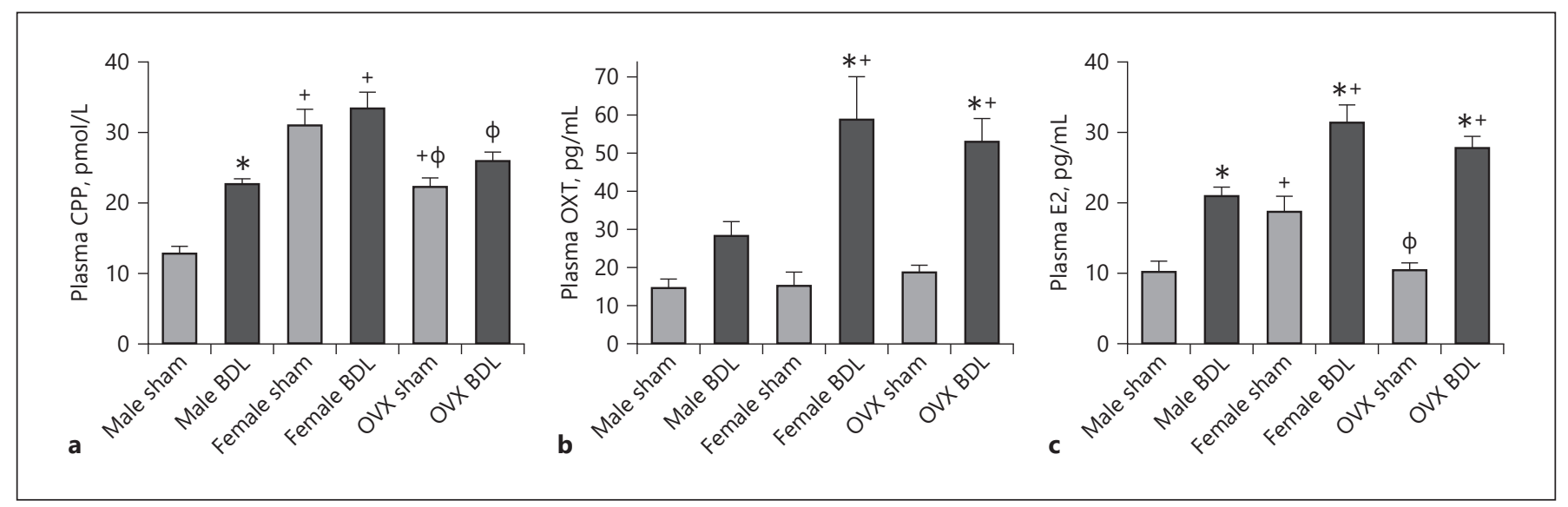

Fig. 5. Effect of BDL in male, female, and OVX rats on plasma CPP concentration (a), plasma OXT concentration (b), and plasma estradiol (E2) concentration (c). Data are mean \pm SEM. ${ }^{*} p<0.05$ vs. respective sham. ${ }^{+} p<$ 0.05 vs. respective male group. ${ }^{\Phi} p<0.05$ vs. respective female group. Groups: male sham $(n=6-7)$; male BDL $(n=6)$; female sham $(n=6-7)$; female BDL $(n=9)$; OVX sham $(n=6-10)$; and OVX BDL $(n=6-10)$.

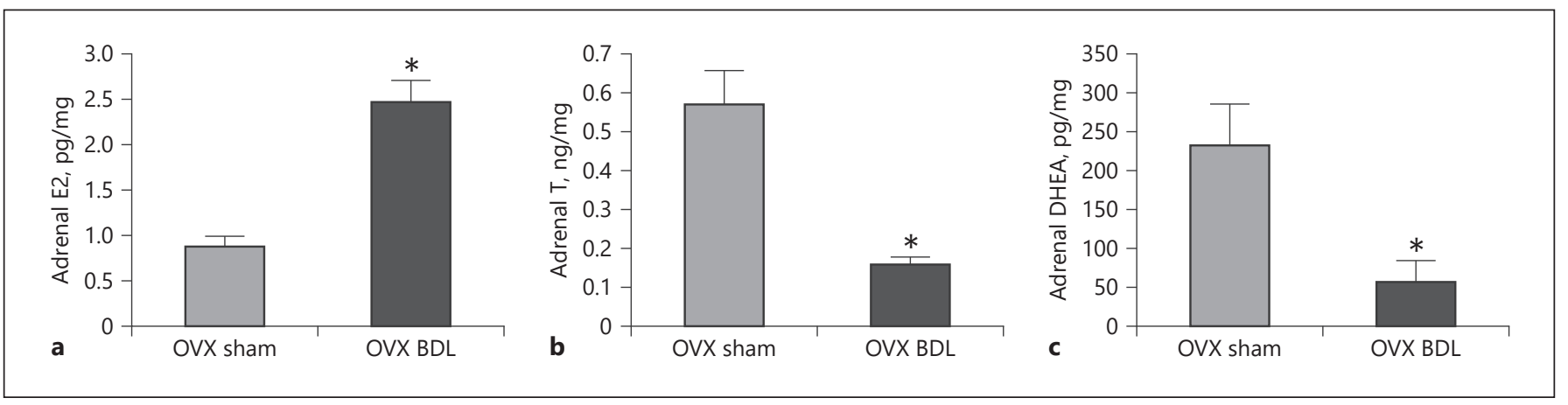

Fig. 6. Effect of BDL and OVX on adrenal glands. Concentrations of adrenal estradiol (a), adrenal testosterone (b), and adrenal DHEA concentration (c). Data are mean \pm SEM. ${ }^{*} p<0.05$ vs. OVX sham. Groups: OVX sham $(n=7)$ and OVX BDL $(n=5)$.

The circulating OXT concentration was significantly increased in OVX BDL rats compared to OVX sham rats (Bonferroni $t=4.293 ; p<0.001$; Fig. $5 b$ ). Similar to female rats, plasma OXT concentration in OVX BDL rats was significantly higher compared to the male BDL group (Bonferroni $t=2.729 ; p<0.05$ ). These data show existence of sex differences in OXT secretion in BDL rats but not in the basal circulating concentration.

\section{Plasma Estradiol Concentration}

Circulating estradiol was measured in plasma samples collected from rats 4 weeks after BDL/sham ligation surgery. Both the treatment and sex factors had significant effects in all BDL rats compared to the sham groups (treatment: $F(1,37)=61.421, p<0.05$; sex: $F(1,37)=9.471$, $p<0.05)$. Plasma estradiol concentration was significant- ly increased in both male and female BDL rats compared to their respective sham groups (male: Bonferroni $t=$ 3.320, $p<0.05$; female: Bonferroni $t=4.084, p<0.05$; Fig. 5c). Also, the estradiol concentration was significantly higher in both the female groups compared to their respective male groups (Bonferroni $t$ tests, all $p<0.05$ ).

Plasma estradiol concentration was measured in the OVX sham and BDL rats to compare the values with intact female sham and BDL rats and verify the efficiency of ovariectomy procedures. Plasma estradiol was significantly reduced in OVX sham rats compared to the intact female sham rats. This indicates the ovariectomy procedures were successful. However, in OVX rats with BDL, circulating estradiol was not reduced. Bonferroni comparisons indicate that plasma estradiol was significantly decreased with ovariectomy in sham rats compared to the 
intact female shams (Bonferroni $t=2.883 ; p<0.05$; Fig. 5c). The OVX BDL rats showed significant increase in circulating estradiol concentration compared to the OVX sham rats (Bonferroni $t=6.561 ; p<0.05$; Fig. $5 c$ ).

\section{Adrenal Steroid Concentration}

Adrenal steroids were extracted to measure the concentrations of estradiol, testosterone, and DHEA. BDL was associated with a greater adrenal estradiol concentration in OVX rats compared to the sham rats. This increase in adrenal estradiol concentration might contribute to the observed increase in circulating estradiol. In contrast, the concentration of estradiol precursors, testosterone and DHEA, were lower in the adrenal glands of OVX BDL rats compared to the OVX sham rats. The lower concentration of the substrates of estradiol synthesis with the greater concentrations of estrogen suggests the possibility of increased enzymatic activity.

Adrenal estradiol concentration in OVX BDL rats was significantly higher than the OVX sham rats $(t=-5.632$; d.f. $=11 ; p<0.001$; Fig. 6a). Adrenal testosterone and DHEA were significantly lower in the OVX BDL rats compared to the OVX sham rats (testosterone: $t=4.685$, d.f. $=11, p<0.001$, Fig. 6b; DHEA: $t=2.783$, d.f. $=11$, $p<0.05$, Fig. $6 c$ ). These data suggest increased aromatase transcription or activity in the adrenal glands.

\section{Discussion/Conclusion}

It has been suggested that cirrhosis and other types of liver disease causes fibrosis, which blocks portal blood flow due to intrahepatic vasoconstriction leading to portal hypertension. Once portal hypertension develops, it influences extrahepatic vascular beds in the splanchnic and systemic circulations resulting in arterial vasodilation [42]. Peripheral vasodilation causes hypovolemia [43]. The decrease in effective blood volume is reported to cause baroreceptor-mediated non-osmotic stimulation of AVP $[1,6,11,15,23]$. In patients with liver cirrhosis, increased AVP secretion leads to hyponatremia, resulting in life-threatening complications such as cerebral and pulmonary edema [2, 14, 44]. Although decreased effective blood volume is the most common explanation for inappropriate AVP release associated with cirrhosis, the mechanism is not known. Other factors such as systemic inflammation could contribute to the changes in body fluid balance related to BDL. Furthermore, no previous studies have reported sex differences in the development of hyponatremia during liver failure.
This study addresses sex differences in development of hyponatremia and AVP secretion in an experimental model of liver cirrhosis. Chronic bile duct-ligated rats were used as an animal model in this study. Cirrhosis was induced in BDL rats by cauterizing the bile duct and obstructing the flow of bile from liver to duodenum. The BDL rats develop jaundice and cirrhosis due to necrosis of hepatocytes caused by bile accumulation in liver. The yellowish pigmentation of skin and plasma, along with an increase in liver to body weight ratio, were used as gross indices of disease progression in this study and previous studies from these groups $[10,36,39,45]$. Although these indices are related to disease progression in this model, they are not clinically reliable indicators such as bilirubin $[33,46]$. Based on these results, it would be worth examining not only bilirubin and possibly other clinical markers of hepatic function.

Plasma CPP measurements were used as an indicator for circulating AVP in our experiments. Plasma CPP is often used as a surrogate marker for AVP as CPP is secreted at equal molar concentration to AVP and has longer half-life compared to AVP. Plasma CPP is known to correlate with AVP measurements at various plasma osmolalities [24, 38, 47].

The results from this study show a distinct sex difference in the development of hyponatremia during liver cirrhosis using the BDL rat model. The stage of the estrous cycle was recorded for intact female rat at the time of sacrifice but did not appear to influence the changes in CPP or OXT measured at the end of the experiment. Unlike male rats, female BDL rats develop liver failure but not hyponatremia. Ovariectomy was performed to understand the influence of circulating estrogen in preventing AVP release in female BDL rats. Plasma estradiol analysis shows that removal of ovaries in BDL rats did not eliminate the estradiol from circulation. We found that adrenal-derived estradiol contributes to the observed increase in circulating plasma estradiol in OVX BDL rats. Similar to the intact female rats, OVX BDL rats developed cirrhosis but did not increase AVP secretion and hyponatremia. The adrenal estradiol might have compensated for the absence of gonadal estradiol preventing OVX BDL rats from becoming hyponatremic.

Plasma estradiol concentrations were higher in all BDL groups compared to their respective shams. Increases in circulating estradiol has been reported in cirrhosis patients $[48,49]$. Elevated estradiol secretion might be protective, as hyponatremia did not develop with increased in estradiol in our experiments. However, the underlying mechanism leading to an increase in estradiol 
and its role in liver failure are not understood. This study attempted to understand the role of estradiol in AVP secretion and hyponatremia in female BDL rats by performing ovariectomy.

Ovariectomy significantly decreased plasma estradiol concentration in sham rats compared to intact female sham ligated rats. However, BDL in OVX rats increased the circulating estradiol concentration. Increased estradiol was observed in intact female and male BDL rats as well. Adrenal glands were collected at the end of protocol to determine a possible source of estradiol in OVX BDL rats. This study is the first to show that BDL increases adrenal estradiol and decreases its precursors in OVX rats compared to the sham rats. This increase in adrenal estradiol concentration might be contributing to the observed increase in circulating estradiol. The decrease in substrates of estradiol synthesis pathway, testosterone and DHEA, and the increase in estradiol suggests the possibility of increased aromatase enzyme activity. Clinical studies show that aromatase inhibitors utilized for treatment of breast cancer induce hepatotoxicity $[50,51]$. Aromatase overexpression has been reported in hepatitis and hepatocellular carcinoma, but the role of aromatase in liver disorders remains unclear. Also, liver cirrhosis in BDL rats might impair estradiol metabolism and possibly contribute to the increased estradiol in circulation. At this point, we cannot address the role of estrogens in the absence of hyponatremia in female BDL rats.

The transcription factor FosB creates a heterodimer with Jun to form activator protein-1 (AP-1) complexes. AP-1 complexes bind with the target gene promoter sequence to either stimulate or inhibit transcription. Our previous study indicates that changes in gene expression associated by increased FosB in the SON may be necessary for increased vasopressin secretion [32]. In other systems, FosB has been shown to contribute to neural sensitization and adaptions in neural function [52-54]. In accordance with the previous literature, male BDL rats had greater numbers of FosB positive neurons in the SON and $61 \%$ of these were positive for AVP. Both the intact female BDL and OVX BDL rats had significantly more FosB-positive neurons in the SON as compared to shamligated controls, but only 24 and 19\%, respectively, were AVP positive. As OXT MNCs are in the SON and PVN along with AVP neurons, this suggests that in female rats the FosB staining was in OXT neurons.

A sex difference was observed in OXT secretion in BDL rats, which could be consistent with the FosB staining. Plasma OXT concentration was higher in intact female BDL and OVX BDL rats compared to female sham.
This increase was not observed in male BDL rats. Studies have reported increases in OXT gene expression associated with increases in plasma estradiol $[55,56]$, but the relationship between estrogens and OXT release is not clear. While estrogen inhibits AVP release in vitro, the same studies show that it inhibits OXT release in vitro as well $[18,21,26]$. Additional studies will be required to determine the mechanisms that increase circulating OXT in female BDL rats.

Both AVP and OXT are metabolized by the liver, and altered hepatic metabolism could contribute to the increases in these hormones that were observed in BDL rats. It was observed that AVP (in this study CPP) was increased in male BDL rats while OXT was increased in BDL female rats. It could be that decreased hepatic metabolism is contributing to increases in AVP and OXT in BDL rats. In male BDL rats, it was previously shown that increased circulating AVP is associated with increased heteronuclear RNA (hnRNA) for AVP in the SON and increased FosB expression. Together, these observations indicate that increased AVP synthesis and release contributes to water retention in male BDL rats. Similar observation about OXT hnRNA have not been made in male BDL rats. It could be that the trend of increased plasma OXT in male $\mathrm{BDL}$ rats is due to changes in the OXT metabolism. In female BDL rats, increased plasma OXT was associated with increased FosB staining in OXT neurons of the SON consistent with increased release. Again, in female BDL rats, there was a trend for increased circulating AVP, and this trend could be due to changes in hepatic metabolism. The mechanisms responsible for OXT and AVP secretion in the BDL model are yet to be understood.

Daily fluid intake and urine excretion were recorded for 14 days beginning after 2 weeks of recovery from $\mathrm{BDL} /$ sham ligation surgery. Fluid intake and urine excretion were greater in BDL rats despite their low plasma osmolalities, suggesting a possible role for non-osmotic mechanisms in drinking behavior of BDL rats. Previous studies from our laboratory have shown that angiotensin type 1 receptors in the subfornical organ and BDNF in the SON contribute to increased fluid intake in male BDL rats $[10,32,45,57]$. Sex differences were observed in fluid intake and urine excretion of BDL rats. The intact female and OVX BDL rats appeared to increase their urine output to compensate for increased average daily water intake as compared to male rats, which might contribute to the observed absence of hyponatremia. Previous studies have shown that $\mathrm{BDL}$ in male rats significantly decreases sodium excretion compared to sham rats [24]. The effects of BDL on sodium excretion may be different in female 
rats because OXT, which was selectively increased in females, can be natriuretic and stimulates atrial natriuretic peptide secretion $[58,59]$. It could be that OXT release by the female BDL rats contributed to their ability to maintain a more normal plasma osmolality. However, changes in blood pressure and sodium excretion or the functional consequences of OXT release have not been tested in female rats and will be addressed in future studies.

Angiotensin II (Ang II) is the main hormone of the renin angiotensin system, and it influences AVP release both as a hormone and a putative neurotransmitter $[60,61]$. In BDL rats, central infusions of losartan decreased water intake but did not prevent the decrease in plasma osmolality associated with BDL [10]. Blocking angiotensin type 1 receptors in the subfornical organ also reduced water intake associated with BDL but, again, had no effect on plasma osmolality or FosB staining in AVP SON neurons [62]. Based on these results, Ang II appears to be more involved in fluid intake than AVP release in male BDL rats. Estrogen influences the peripheral and central effects of Ang II [63, 64], and androgens influence gene expression in the peripheral renin angiotensin system [65]. The role of estrogen in the sex differences that we have observed in BDL rats is still not clear. It could be that testosterone, a combination of estrogen and testosterone, or a different ovarian hormone is primarily responsible for the differences in body fluid balance or neurohypophyseal hormone release or has differential effect on kidney function in BDL rats.

This study advances current understanding of sex differences in the regulation of AVP and OXT related to disorders of body fluid balance associated with cirrhosis. However, the adrenal production of estradiol appeared to confound the use of OVX to determine the role of this hormone in the failure of the female rats to develop hyponatremia during the course of this experiment. It could be that the additional estradiol produced by the adrenal was sufficient to maintain a protective effect of estrogens. Alternatively, the protective mechanism may be unrelated to estrogens such as a chromosomal or organizational effect. Additional experiments are required to understand the role of estradiol in BDL as well as the sex difference re- lated to AVP and OXT release in this model. Expression of ER $\beta$ in MNCs and its possible role in AVP release suggests ER involvement in the prevention of BDL-induced hyponatremia in female rats. Future studies combining ER antagonist in this model of hyponatremia will provide further insight into mechanisms underlying sex differences in hyponatremia related to liver failure.

\section{Acknowledgement}

The authors would like to thank Phong Duong for technical assistance.

\section{Statement of Ethics}

Animal protocols were approved by the UNT Health Science Center IACUC and conducted in accordance to the National Institute of Health Guide for the Care and Use of Laboratory Animals.

\section{Disclosure Statement}

The authors declare that they have no competing interests.

\section{Availability of Data and Materials}

The data sets used and/or analyzed during the current study are available from the corresponding author on reasonable request.

\section{Funding Sources}

This work was supported by R01 HL119458 to J.T.C. and AHA18PRE34060035 to K.B.

\section{Author Contributions}

J.T.C., R.L.C., and K.B. designed experiments; K.B., J.T.L., and M.E.B. performed experiments; K.B., M.E.B., and J.T.C. analyzed data; K.B., R.L.C., and J.T.C. wrote the manuscript. All authors read and approved the final manuscript.

\section{References}

1 Verbalis JG. Disorders of body water homeostasis. Best Pract Res Clin Endocrinol Metab. 2003 Dec;17(4):471-503.

2 John S, Thuluvath PJ. Hyponatremia in cirrhosis: pathophysiology and management. World J Gastroenterol. 2015 Mar;21(11): 3197-205.
3 Hayashi M, Abe K, Fujita M, Okai K, Takahashi A, Ohira H. Association between the Serum Sodium Levels and the Response to Tolvaptan in Liver Cirrhosis Patients with Ascites and Hyponatremia. Intern Med. 2018 Sep;57(17):2451-8.

4 Martin PY, Schrier RW. Sodium and water retention in heart failure: pathogenesis and treatment. Kidney Int Suppl. 1997 Jun; 59:S57-61.

5 Gaglio P, Marfo K, Chiodo J 3rd. Hyponatremia in cirrhosis and end-stage liver disease: treatment with the vasopressin $\mathrm{V}_{2}$-receptor antagonist tolvaptan. Dig Dis Sci. 2012 Nov; 57(11):2774-85. 
6 Cárdenas A, Arroyo V. Mechanisms of water and sodium retention in cirrhosis and the pathogenesis of ascites. Best Pract Res Clin Endocrinol Metab. 2003 Dec;17(4):607-22.

7 Cárdenas A, Ginès P. Pathogenesis and treatment of fluid and electrolyte imbalance in cirrhosis. Semin Nephrol. 2001 May;21(3):30816.

8 Ginès $\mathrm{P}$, Guevara M. Hyponatremia in cirrhosis: pathogenesis, clinical significance, and management. Hepatology. 2008 Sep;48(3): 1002-10.

9 Verbalis JG. Osmotic inhibition of neurohypophysial secretion. Ann N Y Acad Sci. 1993 Jul;689(1):146-60.

10 Walch JD, Carreño FR, Cunningham JT. Intracerebroventricular losartan infusion modulates angiotensin II type 1 receptor expression in the subfornical organ and drinking behaviour in bile-duct-ligated rats. Exp Physiol. 2013 Apr;98(4):922-33.

11 Chatterjee K. Neurohormonal activation in congestive heart failure and the role of vasopressin. Am J Cardiol. 2005 May;95(9A 9a): 8B-13B.

12 Goldsmith SR. Current treatments and novel pharmacologic treatments for hyponatremia in congestive heart failure. Am J Cardiol. 2005 May;95(9A 9a):14B-23B.

13 Aditya S, Rattan A. Vaptans: A new option in the management of hyponatremia. Int J Appl Basic Med Res. 2012 Jul;2(2):77-83.

14 D'Auria D, Marinosci GZ, De Benedictis G, Piazza O. Vaptans and hyponatremia in critical patients. Transl Med UniSa. 2012 Apr 30; 3:1-14.

15 Lo R, Austin A, Freeman J. Vasopressin in liver disease-should we turn on or off? Curr Clin Pharmacol. 2008 Sep;3(3):156-65.

16 Shimizu I, Ito S. Protection of estrogens against the progression of chronic liver disease. Hepatol Res. 2007;37(4):239-47.

17 Shimizu I. Impact of oestrogens on the progression of liver disease. Liver Int. 2003;23(1): 63-9.

18 Sladek CD, Somponpun SJ. Estrogen receptors: their roles in regulation of vasopressin release for maintenance of fluid and electrolyte homeostasis. Front Neuroendocrinol. 2008 Jan;29(1):114-27.

19 Hrabovszky E, Kalló I, Steinhauser A, Merchenthaler I, Coen CW, Petersen SL, et al. Estrogen receptor-beta in oxytocin and vasopressin neurons of the rat and human hypothalamus: immunocytochemical and in situ hybridization studies. J Comp Neurol. 2004 May; 473(3):315-33

20 Sakamoto H, Matsuda K, Hosokawa K, Nishi M, Morris JF, Prossnitz ER, et al. Expression of $\mathrm{G}$ protein-coupled receptor-30, a $\mathrm{G}$ protein-coupled membrane estrogen receptor, in oxytocin neurons of the rat paraventricular and supraoptic nuclei. Endocrinology. 2007 Dec;148(12):5842-50.

21 Sladek CD, Swenson KL, Kapoor R, Sidorowicz HE. The role of steroid hormones in the regulation of vasopressin and oxytocin release and mRNA expression in hypothalamo-neurohypophysial explants from the rat. Exp Physiol. 2000 Mar;85(Spec No s1):171S-7S.

22 Bourque CW. Central mechanisms of osmosensation and systemic osmoregulation. Nat Rev Neurosci. 2008 Jul;9(7):519-31.

23 Gizowski C, Bourque CW. The neural basis of homeostatic and anticipatory thirst. Nat Rev Nephrol. 2018 Jan;14(1):11-25.

24 Balapattabi K, Farmer GE, Knapp BA, Little JT, Bachelor M, Yuan JP, et al. Effects of saltloading on supraoptic vasopressin neurones assessed by ClopHensorN chloride imaging. J Neuroendocrinol. 2019 Aug;31(8):e12752.

25 Balapattabi K, Little JT, Farmer GE, Cunningham JT. High salt loading increases brain derived neurotrophic factor in supraoptic vasopressin neurones. J Neuroendocrinol. 2018 Nov;30(11):e12639.

26 Somponpun S, Sladek CD. Role of estrogen receptor-beta in regulation of vasopressin and oxytocin release in vitro. Endocrinology. 2002 Aug;143(8):2899-904.

27 Shughrue PJ, Komm B, Merchenthaler I. The distribution of estrogen receptor-beta mRNA in the rat hypothalamus. Steroids. 1996 Dec; 61(12):678-81.

28 Suzuki S, Handa RJ. Estrogen receptor-beta, but not estrogen receptor-alpha, is expressed in prolactin neurons of the female rat paraventricular and supraoptic nuclei: comparison with other neuropeptides. J Comp Neurol. 2005 Mar;484(1):28-42.

29 Share L, Crofton JT, Ouchi Y. Vasopressin: sexual dimorphism in secretion, cardiovascular actions and hypertension. Am J Med Sci. 1988 Apr;295(4):314-9.

30 Crowley WR, O'Donohue TL, George JM, Jacobowitz DM. Changes in pituitary oxytocin and vasopressin during the estrous cycle and after ovarian hormones: evidence for mediation by norepinephrine. Life Sci. 1978 Dec; 23(26):2579-85.

31 Greer ER, Caldwell JD, Johnson MF, Prange AJ Jr, Pedersen CA. Variations in concentration of oxytocin and vasopressin in the paraventricular nucleus of the hypothalamus during the estrous cycle in rats. Life Sci. 1986 Jun; 38(25):2311-8.

32 Cunningham JT, Nedungadi TP, Walch JD, Nestler EJ, Gottlieb HB. $\triangle$ FosB in the supraoptic nucleus contributes to hyponatremia in rats with cirrhosis. Am J Physiol Regul Integr Comp Physiol. 2012 Jul;303(2):R177-85.

33 Liu Y, Meyer C, Xu C, Weng H, Hellerbrand C, ten Dijke P, et al. Animal models of chronic liver diseases. Am J Physiol Gastrointest Liver Physiol. 2013 Mar;304(5):G449-68.

34 Zou W. Ovariectomy (oophorectomy). Protocol Exchange. DOI: 10.1038/protex.2011. 242.

35 Khajuria DK, Razdan R, Mahapatra DR. Descrição de um novo método de ooforectomia em ratas. Rev Bras Reumatol. 2012;52(3): 466-70.

36 Carreño FR, Ji LL, Cunningham JT. Altered central TRPV4 expression and lipid raft as- sociation related to inappropriate vasopressin secretion in cirrhotic rats. Am J Physiol Regul Integr Comp Physiol. 2009 Feb;296(2):R45466.

37 Cora MC, Kooistra L, Travlos G. Vaginal Cytology of the Laboratory Rat and Mouse: Review and Criteria for the Staging of the Estrous Cycle Using Stained Vaginal Smears. Toxicol Pathol. 2015 Aug;43(6):776-93.

38 Balanescu S, Kopp P, Gaskill MB, Morgenthaler NG, Schindler C, Rutishauser J. Correlation of plasma copeptin and vasopressin concentrations in hypo-, iso-, and hyperosmolar States. J Clin Endocrinol Metab. 2011 Apr;96(4):1046-52.

39 Nedungadi TP, Cunningham JT. Differential regulation of TRPC4 in the vasopressin magnocellular system by water deprivation and hepatic cirrhosis in the rat. Am J Physiol Regul Integr Comp Physiol. 2014 Mar; 306(5):R304-14

40 Rosario ER, Chang L, Head EH, Stanczyk FZ, Pike CJ. Brain levels of sex steroid hormones in men and women during normal aging and in Alzheimer's disease. Neurobiol Aging. 2011 Apr;32(4):604-13.

41 Choe KY, Han SY, Gaub P, Shell B, Voisin DL, Knapp BA, et al. High salt intake increases blood pressure via BDNF-mediated downregulation of KCC2 and impaired baroreflex inhibition of vasopressin neurons. Neuron. 2015 Feb;85(3):549-60.

42 Iwakiri Y. Pathophysiology of portal hypertension. Clin Liver Dis. 2014 May;18(2):281-91.

43 Møller S, Bendtsen F. The pathophysiology of arterial vasodilatation and hyperdynamic circulation in cirrhosis. Liver Int. 2018;38(4): 570-80.

44 Kleindienst A, Brabant G, Morgenthaler NG, Dixit KC, Parsch H, Buchfelder M. Following brain trauma, copeptin, a stable peptide derived from the AVP precusor, does not reflect osmoregulation but correlates with injury severity. Acta Neurochir Suppl (Wien). 2010; 106:221-4.

45 Saxena A, Bachelor M, Park YH, Carreno FR, Nedungadi TP, Cunningham JT. Angiotensin II induces membrane trafficking of natively expressed transient receptor potential vanilloid type 4 channels in hypothalamic $4 \mathrm{~B}$ cells. Am J Physiol Regul Integr Comp Physiol. 2014 Oct;307(8):R945-55.

46 Kountouras J, Billing BH, Scheuer PJ. Prolonged bile duct obstruction: a new experimental model for cirrhosis in the rat. Br J Exp Pathol. 1984 Jun;65(3):305-11.

47 Santillan MK, Santillan DA, Scroggins SM, Min JY, Sandgren JA, Pearson NA, et al. Vasopressin in preeclampsia: a novel very early human pregnancy biomarker and clinically relevant mouse model. Hypertension (Dallas). 2014;64(4):852-9.

48 Baker HW, Burger HG, de Kretser DM, Dulmanis A, Hudson B, O'Connor S, et al. A study of the endocrine manifestations of hepatic cirrhosis. Q J Med. 1976 Jan;45(177): 145-78. 
49 Zumoff B, Fishman J, Gallagher TF, Hellman L. Estradiol metabolism in cirrhosis. J Clin Invest. 1968 Jan;47(1):20-5.

50 Hata S, Miki Y, Saito R, Ishida K, Watanabe $\mathrm{M}$, Sasano H. Aromatase in human liver and its diseases. Cancer Med. 2013 Jun;2(3):30515.

51 Lee JI, Yu JH, Anh SG, Lee HW, Jeong J, Lee KS. Aromatase Inhibitors and Newly Developed Nonalcoholic Fatty Liver Disease in Postmenopausal Patients with Early Breast Cancer: A Propensity Score-Matched Cohort Study. Oncologist. 2019 Aug;24(8):e653-61.

52 Chen J, Kelz MB, Hope BT, Nakabeppu Y, Nestler EJ. Chronic Fos-related antigens: stable variants of deltaFosB induced in brain by chronic treatments. J Neurosci. 1997 Jul; 17(13):4933-41.

53 Kelz MB, Nestler EJ. deltaFosB: a molecular switch underlying long-term neural plasticity. Curr Opin Neurol. 2000 Dec;13(6):715-20.

54 McClung CA, Ulery PG, Perrotti LI, Zachariou V, Berton O, Nestler EJ. DeltaFosB: a molecular switch for long-term adaptation in the brain. Brain Res Mol Brain Res. 2004 Dec; 132(2):146-54.
55 McCarthy MM. Estrogen modulation of oxytocin and its relation to behavior. Adv Exp Med Biol. 1995;395:235-45.

56 Chung SK, McCabe JT, Pfaff DW. Estrogen influences on oxytocin mRNA expression in preoptic and anterior hypothalamic regions studied by in situ hybridization. J Comp Neurol. 1991 May;307(2):281-95.

57 Balapattabi K, Little J, Bachelor M, Cunningham J. Brain Derived Neurotrophic Factor and Supraoptic Vasopressin Neurons in Hyponatremia. Neuroendocrinology. 2019 Sep. https://doi.org/10.1159/000503723.

58 Soares TJ, Coimbra TM, Martins AR, Pereira AG, Carnio EC, Branco LG, et al. Atrial natriuretic peptide and oxytocin induce natriuresis by release of cGMP. Proc Natl Acad Sci USA. 1999 Jan;96(1):278-83.

59 Gutkowska J, Jankowski M, Lambert C, Mukaddam-Daher S, Zingg HH, McCann SM. Oxytocin releases atrial natriuretic peptide by combining with oxytocin receptors in the heart. Proc Natl Acad Sci USA. 1997 Oct; 94(21):11704-9.

60 McKinley MJ, Yao ST, Uschakov A, McAllen RM, Rundgren M, Martelli D. The median preoptic nucleus: front and centre for the regulation of body fluid, sodium, temperature, sleep and cardiovascular homeostasis. Acta Physiol (Oxf). 2015 May;214(1):8-32.
61 McKinley MJ, Mathai ML, McAllen RM, McClear RC, Miselis RR, Pennington GL, et al. Vasopressin secretion: osmotic and hormonal regulation by the lamina terminalis. J Neuroendocrinol. 2004 Apr;16(4):340-7.

62 Walch JD, Nedungadi TP, Cunningham JT. ANG II receptor subtype la gene knockdown in the subfornical organ prevents increased drinking behavior in bile duct-ligated rats. Am J Physiol Regul Integr Comp Physiol. 2014 Sep;307(6):R597-607.

63 O'Donnell E, Floras JS, Harvey PJ. Estrogen status and the renin angiotensin aldosterone system. Am J Physiol Regul Integr Comp Physiol. 2014 Sep;307(5):R498-500.

64 Xue B, Johnson AK, Hay M. Sex differences in angiotensin II- and aldosterone-induced hypertension: the central protective effects of estrogen. Am J Physiol Regul Integr Comp Physiol. 2013 Sep;305(5):R459-63.

65 Fischer M, Baessler A, Schunkert H. Renin angiotensin system and gender differences in the cardiovascular system. Cardiovasc Res. 2002 Feb;53(3):672-7. 